Bundesgesundheitsbl 2022 · 65:58-66 https://doi.org/10.1007/s00103-021-03468-w Eingegangen: 30. August 2021

c c Der/die Autor(en) 2021

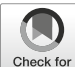

Angenommen: 26. November 2021

Online publiziert: 30. Dezember 2021

\author{
Barbara Schneider ${ }^{1,2} \cdot$ Andreas Reif $^{1} \cdot$ Birgit Wagner $^{3} \cdot$ Manfred Wolfersdorf $^{4}$ \\ ${ }^{\prime}$ Klinik für Psychiatrie, Psychosomatik und Psychotherapie, Johann Wolfgang-Goethe-Universität \\ Frankfurt/Main, Frankfurt/Main, Deutschland \\ ${ }^{2}$ Abteilung für Abhängigkeitserkrankungen, Psychiatrie und Psychotherapie, LVR-Klinik Köln, Köln, \\ Deutschland \\ ${ }^{3}$ MSB Medical School Berlin, Berlin, Deutschland \\ ${ }^{4}$ Universität Bayreuth, Bayreuth, Deutschland
}

\section{Warum brauchen wir Leitlinien für Suizidprävention?}

\section{Einleitung}

In Deutschland nehmen sich jährlich mehr als 9000 Menschen das Leben (2019: 9041 Suizide - davon 6842 Männer und 2199 Frauen [1] - entsprechend einer Suizidrate von 11,6/100.000 Einwohner). In Mitteleuropa steigt mit zunehmendem Lebensalter die Suizidrate an, insbesondere bei den Männern. Es wird davon ausgegangen, dass jeder Suizid im Mittel 8-10 weitere Menschen unmittelbar in Mitleidenschaft zieht [2], also pro Jahr weitere ca. 70.000-90.000. Bei jüngeren Menschen ist Suizid eine der häufigsten Todesursachen. Es gibt bereits in der 4. Überarbeitung eine deutsche Leitlinie der Arbeitsgemeinschaft der Wissenschaftlichen Medizinischen Fachgesellschaften (AWMF) zum Thema Suizidalität im Kindes- und Jugendalter [3], die unter Federführung der Deutschen Gesellschaft für Kinderund Jugendpsychiatrie, Psychosomatik und Psychotherapie e.V. (DGKJP) entstanden ist. Derzeit steht die fünfte Aktualisierung dieser Leitlinie an. Eine Leitlinie zum Umgang mit Suizidalität im Erwachsenenalter gibt es bisher nicht. Im August 2021 wurde jedoch die Finanzierung einer S3-Leitlinie „Umgang mit Suizidalität" vom Innovationsfonds des Gemeinsamen Bundesausschusses bewilligt.

In Deutschland unterstützt und fördert die AWMF die Entwicklung von Leitlinien u. a. zur Behandlung von psychischen und somatischen Erkrankungen. Im Jahr 1995 hat der Sachverstän- digenrat für die Konzertierte Aktion im Gesundheitswesen die AWMF erstmalig beauftragt, die Entwicklung von Standards, Richtlinien, Leitlinien und Empfehlungen der wissenschaftlichen medizinischen Fachgesellschaften zu koordinieren. Inzwischen sind in Deutschland insgesamt mehr als 800 Leitlinien erschienen. Es besteht jedoch bisher keine dezidierte deutschsprachige Leitlinie für den Umgang mit Suizidalität bei Erwachsenen, obwohl suizidales Verhalten ein wesentlicher Faktor von Mortalität und Morbidität ist und auch ein großes gesellschaftliches Problem darstellt.

Im Vereinigten Königreich existiert die englischsprachige (evidenzbasierte) Leitlinie des National Institute for Health and Care Excellence (NICEGuideline) „Suicide prevention“ (letzte Überarbeitung 2018 [4]). Aufgrund der unterschiedlichen Versorgungssysteme lässt sie sich jedoch nur schwer auf die deutsche Situation anwenden und übertragen. Darüber hinaus unterscheidet sich die zugrunde liegende NICEMethodologie grundsätzlich von der AWMF-S3-Methodik, die sowohl evidenzbasiert als auch konsensorientiert ist $[5,6]$. Auch außerhalb der psychiatrischpsychotherapeutischen Versorgung hat suizidales Verhalten eine hohe Relevanz, in der hausärztlichen Versorgung, aber vor allem in der Neurologie, der Chirurgie, der Allgemeinmedizin/Inneren Medizin einschließlich der Geriatrie. Insbesondere muss im Zusammenhang mit onkologischen und palliativmedizinischen Fragestellungen an Suizidalität gedacht werden. Suizidversuche führen häufig zur Inanspruchnahme des Rettungsdienstes, der Notaufnahmen und zu intensivmedizinischer Versorgung. Die entsprechenden (Weiter-)Behandlungsketten, z. B. Vermittlung von fachpsychiatrischer Evaluation einer zugrunde liegenden psychischen Erkrankung und spezifische Behandlung des suizidalen Syndroms, sind jedoch nicht standardisiert und es kommt sowohl (häufig) zu Unter- als auch (seltener) zu Überversorgung. Die Qualifikation im Hinblick auf den Umgang mit suizidalem Verhalten ist sehr heterogen; da bislang keine Leitlinie existiert, besteht hier häufig Unklarheit im Hinblick auf das weitere Vorgehen und somit eine erhebliche Versorgungslücke.

\section{Grundbegriffe und epidemiologische Grundlagen}

Es gibt eine Vielzahl von Definitionen von Suizidalität. Die Weltgesundheitsorganisation (WHO; [7]) wählte eine pragmatische Definition, die Suizidgedanken, Suizidabsichten, Suizidpläne, Suizidversuche und Suizide umfasst. Unter dem Begriff "Suizidalität" werden somit alle Gefühle, Gedanken, Impulse und Handlungen zusammengefasst, die selbstzerstörerischen Charakter haben und das eigene Versterben aktiv oder durch Unterlassung anstreben bzw. direkt oder indirekt in Kauf nehmen. Im wissenschaftlichen Sprachgebrauch wird grundsätzlich der wertfreie Begriff ,Suizid“ und nicht die wertenden Begriffe 
„Selbstmord“ oder „Freitod“ verwendet. Aktuelle, umfassende Übersichtsarbeiten zum Thema Suizidalität wurden in den letzten Jahren von Turecki und Brent [8] und Fazel und Runeson [9] vorgelegt.

Suizidversuche als solche werden im Gegensatz zu vollendeten Suiziden in Deutschland nicht systematisch erfasst, weisen eine hohe Dunkelziffer auf und können lediglich durch Stichprobenhochrechnungen ermittelt werden. Nach Einschätzung der WHO [7] ereignen sich jährlich 10 - bis 30 -mal so viele Suizidversuche wie Suizide. Demnach kann man in Deutschland von geschätzt 100.000 bis hin zu 300.000 Suizidversuchen ausgehen. Eine signifikante Anzahl hiervon (ca. 20-30\%) führt zur Inanspruchnahme von medizinischen Versorgungsleistungen unterschiedlicher Art [1]. Die fehlende Erfassung von Suizidversuchen ist daher ein Problem, weil vorangegangene Suizidversuche einen der wichtigsten Risikofaktoren für den vollendeten Suizid darstellen $[10,11]$, sodass die Chance zur individuellen Sekundärprävention vertan wird. Aber auch säkulare Trends im Hinblick auf Methoden oder Orte werden deshalb übersehen, was präventives Eingreifen erschwert.

In Mitteleuropa steigt mit zunehmendem Lebensalter die Suizidrate an, insbesondere jedoch bei den Männern [1]. Die Suizidprävention im Alter ist eine besondere Herausforderung, was durch den demografischen Wandel in den kommenden Jahren weiter verstärkt wird. Hinzu kommt, dass in Deutschland eine Neuregelung eines Gesetzes zum assistierten Suizid nach dem Urteil des Bundesverfassungsgerichts (BVerfG) vom 26.02.2020 ansteht [12]; in fast allen Ländern mit einer liberalen Gesetzgebung zum assistierten Suizid steigen die Zahlen der eigenständigen Suizide an, (z. B. [13], siehe [14]), sodass davon ausgegangen werden muss, dass auch in Deutschland die Zahl der Suizide zunimmt, falls eine Liberalisierung der Gesetzgebung zum assistierten Suizid eintreten würde.

Meist, aber nicht immer liegt bei einem Suizid eine schwere psychische Erkrankung vor; hinzu kommen meist endogen oder exogen getriggerte, krisenhafte Zuspitzungen als hinreichende Fak- toren. Suizidalität ist für das Individuum (und seine Angehörigen) sehr belastend und hat negative psychische, soziale und körperliche Folgen. Suizidalität entsteht im Zusammenspiel von individuellbiografischen, somatisch-gesundheitlichen und gesellschaftlich-kulturellen Einflussfaktoren in unterschiedlicher Gewichtung sowie aktuellen Lebensereignissen und ist somit ein komplexes Phänomen. Suizidalität als Phänomen ist meist vorübergehend, Suizid jedoch endgültig, was die Notwendigkeit von adäquatem, evidenzbasiertem Umgang mit Suizidalität aufzeigt.

Mit dem höchsten Suizidrisiko ist das Vorliegen einer psychischen Erkrankung assoziiert - bei bis zu $90 \%$ aller vollendeten Suizide lässt sich eine solche prämortal nachweisen -, jedoch kann dennoch nicht automatisch von Suizidalität auf das Vorliegen einer psychischen Erkrankung geschlossen werden [15]; selbst bei einem vollendeten Suizid liegt insbesondere bei jungen Männern oft keine psychiatrische Diagnose vor [16].

Bei beinahe allen psychischen Erkrankungen ist das Suizidrisiko deutlich erhöht [17], insbesondere jedoch bei affektiven Erkrankungen (Depression, bipolare Störung), Alkohol- und Drogenabhängigkeit, schizophrenen Psychosen, Persönlichkeitsstörungen und auch Schlafstörungen [18-21]. Die vorliegenden Metaanalysen sowie auch neuere Untersuchungen zeigen, dass an affektiven Störungen Erkrankte ein etwa 20fach erhöhtes Suizidrisiko im Vergleich zur restlichen Bevölkerung haben [18, 19, 22]. Bei Personen mit Alkoholmissbrauch und -abhängigkeit besteht ein fast 6-fach erhöhtes Suizidrisiko, wobei das Suizidrisiko bei Frauen stärker erhöht ist [20]. Patientinnen und Patienten, die an mehr als einer psychischen Erkrankung (Komorbidität) leiden, haben ein besonders stark erhöhtes Suizidrisiko [23].

Körperliche Erkrankungen wie bösartige Tumorerkrankungen, Niereninsuffizienz, Schlaganfall und andere neurologische Erkrankungen sind ebenfalls Risikofaktoren für Suizid [24-28]. Zudem sind verschiedene soziodemografische Faktoren wie Unverheiratetsein, Alleineleben und LGBTQI+ (lesbisch, schwul, bise- xuell, trans, queer, intersexuell und weitere Geschlechtsidentitäten) Risikofaktoren für Suizid [29-31]. Auch Migration ist mit Suizidalität assoziiert, insbesondere mit Suizidversuchen [32]. Als ein weiterer Risikofaktor gilt Suizidalität in der Familienanamnese [33].

\section{Hintergrund: Geschichte der Leitlinienentwicklung in Deutschland}

In Deutschland sind in der AWMF 180 wissenschaftliche Fachgesellschaften aus allen Bereichen der Medizin zusammengeschlossen. Die AWMF hat das AWMFRegelwerk zur Erstellung und Publikation aktueller und hochwertiger Leitlinien der wissenschaftlichen medizinischen Fachgesellschaften im AWMF-Leitlinienregister erstellt. Darüber hinaus gibt es seit 2003 die Initiative der Nationalen Versorgungsleitlinien (NVL), an der neben der AWMF auch die Bundesärztekammer und die Kassenärztliche Bundesvereinigung beteiligt sind. Die NVL wird obligat vom Ärztlichen Zentrum für Qualität in der Medizin (ÄZQ) und nicht von Fachgesellschaften koordiniert. Die einzige NVL im Bereich der Psychiatrie betrifft das Krankheitsbild der unipolaren Depression. Im Unterschied zu den Leitlinien, die unter Federführung der AWMF erstellt sind, beinhalten die Nationalen Versorgungsleitlinien auch immer eine Patientenleitlinie, welche sich direkt an Patientinnen und Patienten richtet.

Leitlinien stellen den nach einem definierten, transparent gemachten Vorgehen erzielten Konsens mehrerer Expertinnen und Experten aus unterschiedlichen Fachbereichen und Arbeitsgruppen (möglichst unter Einbeziehung von anderen Fachberufen des Gesundheitswesens und Patientinnen und Patienten) zu medizinischen Vorgehensweisen dar. Der Konsens richtet sich nach der wissenschaftlichen Evidenz, wobei Metaanalysen u. Ä. die größte Bedeutung zukommt. Lediglich für Bereiche, bei denen es keinerlei kontrollierte Studien gibt, wird ein klinischer Konsenspunkt formuliert. Leitlinien richten sich vor allem an Ärztinnen und Ärzte, aber auch an Pflegekräfte, psychologische Psychothe- 
rapeutinnen und Psychotherapeuten und andere Fachleute im Gesundheitswesen.

\section{Warum arbeiten wir mit Leitlinien?}

Leitlinien sind systematisch entwickelte, wissenschaftlich begründete und praxisorientierte Entscheidungshilfen für die angemessene medizinische Vorgehensweise bei gesundheitlichen Problemen. Vorrangiges Ziel von Leitlinien ist die Verbesserung der Qualität medizinischer Versorgung durch Wissensvermittlung auf dem neuesten Stand der Wissenschaft im Sinn der evidenzbasierten Medizin. Einerseits werden Empfehlungen abgegeben für Maßnahmen, die evidenzbasiert und unter Abwägung des RisikoNutzen-Profils effektiv sind; andererseits wird von Maßnahmen abgeraten, für die in Studien kein Wirksamkeitsnachweis geführt werden konnte oder bei denen Nichtwirksamkeit oder sogar ein negativer Effekt nachgewiesen wurde. Leitlinien haben die Aufgabe, die wissenschaftliche Evidenz zu speziellen medizinischen Fragestellungen auf dem Boden der klinischen Erfahrung explizit darzulegen, unter methodischen und klinischen Aspekten zu bewerten, gegensätzliche Standpunkte zu klären sowie unter Abwägung von Nutzen und Schaden das derzeitige Vorgehen der Wahl zu definieren. Im Gegensatz zu Richtlinien sind sie rechtlich nicht verbindlich. Das heißt, Ärztinnen und Ärzte und psychologische Psychotherapeutinnen und Psychotherapeuten können von der in der Leitlinie empfohlenen Behandlung abweichen, wenn sie denken, dass die dargestellten Empfehlungen für bestimmte Patientinnen und Patienten nicht geeignet sind. Abweichungen sollten aber jeweils begründet sein. Leitlinien werden regelmäßig aktualisiert; aktuell diskutiert wird eine laufende Aktualisierung im Sinne einer "living guideline“, was insbesondere auf Gebieten mit sich rasch ändernden Empfehlungen (bspw. Onkologie) sehr sinnvoll scheint. Hierbei sind digitale Lösungen, wie beispielswei-

Bundesgesundheitsbl 2022 $65: 58-66$ https://doi.org/10.1007/s00103-021-03468-w ( ) Der/die Autor(en) 2021

\section{B. Schneider · A. Reif $\cdot$ B. Wagner $\cdot$ M. Wolfersdorf}

\section{Warum brauchen wir Leitlinien für Suizidprävention?}

\section{Zusammenfassung}

Trotz der Relevanz des Themas Suizidalität und gut bekannter Risikofaktoren gibt es bisher keine deutsche Leitlinie zur Suizidalität im Erwachsenenalter. In diesem Beitrag werden zunächst die Geschichte und die Hintergründe der Arbeit mit Leitlinien beschrieben. Der aktuelle Stand der Leitlinien für psychische Erkrankungen in Deutschland wird dargestellt und auf suizidpräventive Inhalte hin untersucht. Die Notwendigkeit evidenzbasierter Suizidprävention und einer spezifischen Leitlinie zur Suizidprävention bei Erwachsenen wird diskutiert.

Nur durch gezielte Suizidpräventionsstrategien und Interventionen für die jeweiligen Risikogruppen und unter Beachtung von Alters- und Geschlechtsspezifität kann für alle Betroffenen eine flächendeckende, gut erreichbare, bedarfs- und versorgungsgerechte, finanzierbare sowie nachhaltige medizinische Versorgung auf einem hohen Niveau sichergestellt werden. Dies gilt für den ambulanten und den stationären Bereich sowie für deren Schnittstellen. Bei Suizidalität handelt es sich um ein diagnoseübergreifendes, in unterschiedlichen Versorgungskontexten auftretendes Syndrom mit komplexem Behandlungsbedarf, weshalb intersektorale und multiprofessionelle Aspekte in einer entsprechenden Leitlinie besonders zu adressieren sind. Wissenschaftliche Evidenz und interdisziplinärer Konsens unter Expertinnen und Experten zum Umgang mit suizidalem Verhalten in der medizinischen Versorgung können dazu beitragen, Morbidität und Mortalität im Zusammenhang mit Suizidalität zu reduzieren. Im August 2021 wurde die Finanzierung einer S3-Leitlinie "Umgang mit Suizidalität" vom Innovationsfonds des Gemeinsamen Bundesausschusses bewilligt.

Schlüsselwörter

Suizidalität · Postvention · Leitlinien . Evidenzbasierung $\cdot$ Konsens

\section{Why do we require clinical guidelines for suicide prevention?}

\section{Abstract}

At present, there are no German guidelines regarding suicidal behaviors in adulthood despite their relevance to public health and the well-established evidence about their prevention. This paper first describes the history and background of working with guidelines. The current status of guidelines for mental illness in Germany is presented and examined for suicide-preventive content. The need for evidence-based suicide prevention and a specific guideline for suicide prevention in adults is discussed.

Only via targeted suicide prevention strategies and interventions for the respective risk groups, and by paying particular attention to age and gender specificity in the outpatient as well as inpatient sector, can a high level of care for all patients be ensured. Such strategies have to pay specific attention to the interface between the individual care sectors and need to take comprehensive, easily accessible, needs-based, and affordable sustainable medical care into account. This applies to the outpatient and inpatient sectors as well as to their interfaces. Suicidality is a cross-diagnosis syndrome that occurs in different care contexts and requires complex treatment; therefore, intersectoral and multiprofessional aspects must particularly be addressed in the guideline. Scientific evidence and interdisciplinary expert consensus on the management of suicidal behavior in medical care can help reduce morbidity and mortality associated with suicidality. In August 2021, the funding of an S3 guideline "Management of Suicidality" was approved by the Federal Joint Committee.

Keywords

Suicidality · Postvention - Guidelines . Evidence-based $\cdot$ Consensus

\footnotetext{
1 (https://app.magicapp.org/).
} 
Tab. 1 Aktueller Stand der veröffentlichten Leitlinien im Bereich Psychiatrie unter Federführung der DGPPNund/oder DGKJP (siehe auch [5, 35]), ergänzt um das Ergebnis der Suche nach dem Stichwort "Suizid"

Leitlinien unter DGPPN und/oder DGKJP-Federführung

\begin{tabular}{l|l|}
$\begin{array}{l}\text { AWMF- } \\
\text { Register- } \\
\text { nummer }\end{array}$ & \\
\hline
\end{tabular}

Berücksichtigung der Suizidalität auf jeden Fall erforderlich

Aufmerksamkeitsdefizit-Hyperaktivitätsstörung (ADHS) bei Kindern, Jugendlichen und Erwachsenen

Autismus-Spektrum-Störungen im Kindes-, Jugend- und Erwachsenenalter, Teil 1: Diagnostik

Autismus-Spektrum-Störungen im Kindes-, Jugend- und Erwachsenenalter, Teil 2: Therapie

Begutachtung psychischer und psychosomatischer Erkrankungen Bipolare Störungen

\begin{tabular}{|l|l|l|}
\hline $028-045$ & S3 & Gültig \\
\hline $028-018$ & S3 & Gültig \\
\hline $028-047$ & S3 & Gültig \\
\hline $051-029$ & S2k & Gültig \\
\hline $038-019$ & S3 & Gültig \\
\hline
\end{tabular}

Demenzen

Diagnostik und Behandlung von Zwangsstörungen im Kindesund Jugendalter

Einwilligung bei Demenzen

Essstörungen, Diagnostik und Therapie

Intelligenzminderung

Medikamentenbezogene Störungen

Metamphetamin bezogene Störungen

Nichtorganische Schlafstörungen (F51)

Notfallpsychiatrie

Psychosoziale Therapien bei schweren psychischen Erkrankungen

Rauchen und Tabakabhängigkeit: Screening, Diagnostik und Behandlung

Schizophrenie

Screening, Diagnose und Behandlung alkoholbezogener Störungen

Verhinderung von Zwang: Prävention und Therapie aggressiven Verhaltens bei Erwachsenen

Kindesmisshandlung, -missbrauch, -vernachlässigung unter Einbindung der Jugendhilfe und Pädagogik (Kinderschutzleitlinie)

Behandlung von depressiven Störungen bei Kindern und Jugendlichen

Enuresis und nichtorganische (funktionelle) Harninkontinenz bei Kindern und Jugendlichen

Zwangsstörungen

Suizidalität im Kindes- und Jugendalter

Nichtsuizidales Selbstverletzendes Verhalten (NSSV) im Kindesund Jugendalter

Autonomieförderung und Prävention von Zwangsmaßnahmen, Unterbringungen und freiheitsentziehenden Maßnahmen in der kinder- und jugendpsychiatrischen und -psychotherapeutischen Behandlung

Behandlung Cannabis bezogener Störungen

\begin{tabular}{|c|c|c|c|}
\hline & & & \\
\hline 038-013 & S3 & Gültig & - \\
\hline 028-007 & S3 & Gültig & 10-mal erwähnt \\
\hline $108-001$ & S2k & Gültig & $\begin{array}{l}\text { Erscheint nur im Literaturverzeich- } \\
\text { nis }\end{array}$ \\
\hline $051-026$ & S3 & Gültig & 22-mal erwähnt \\
\hline 028-042 & S2k & Gültig & 2-mal erwähnt \\
\hline 038-025 & S3 & Gültig & 8-mal erwähnt \\
\hline 038-024 & S3 & Gültig & 10-mal erwähnt \\
\hline 028-012 & S1 & Gültig & 2-mal erwähnt \\
\hline 038-023 & S2k & Gültig & Eigenes Kapitel über Suizid \\
\hline 038-020 & S3 & Gültig & 20-mal erwähnt \\
\hline 076-006 & S3 & Gültig & 11-mal erwähnt \\
\hline 038-009 & S3 & Gültig & Eigenes Kapitel über Suizidalität \\
\hline 076-001 & S3 & Gültig & 12-mal erwähnt \\
\hline 038-022 & S3 & Gültig & 11-mal erwähnt \\
\hline 027-069 & S3 & Gültig & 5-mal erwähnt \\
\hline 028-043 & S3 & In Überarbeitung & 48-mal erwähnt \\
\hline $028-026$ & S2k & In Überarbeitung & - \\
\hline 038-017 & S3 & In Überarbeitung & 3-mal erwähnt \\
\hline 028-031 & S2k & $\begin{array}{l}\text { Anmeldeverfahren für } \\
\text { Überarbeitung noch } \\
\text { nicht abgeschlossen }\end{array}$ & Eigene Leitlinie für Suizidalität \\
\hline 028-029 & S2k & Abgelaufen & $\begin{array}{l}\text { Leitlinie dient zur Abgrenzung } \\
\text { gegenüber Suizidalität }\end{array}$ \\
\hline 028-048 & S2k & Angemeldet & N. z. \\
\hline 076-005 & S3 & Angemeldet & N.z. \\
\hline
\end{tabular}

\section{Suizidalität erwähnt}

3-mal erwähnt

3-mal erwähnt (u. a. als Aspekt, den die Anamneseerhebung beinhalten soll)

Empfehlung 90: C.7.4.12 Umgang mit Suizidalität (konsensbasiert)

2-mal erwähnt

Deutsche Gesellschaft für Suizidprävention ist beteiligt

Gesondertes ausführliches Kapitel hierzu; ein Empfehlungsgrad A und ein Empfehlungsgrad $B$

14 Empfehlungen zur Suizidalität

Erscheint nur im Literaturverzeichnis

2-mal erwähnt

2-mal erwähnt

Kapitel über Suizid

Eigenes Kapitel über Suizidalität 


\begin{tabular}{|c|c|c|c|c|}
\hline Leitlinien unter DGPPN und/oder DGKJP-Federführung & $\begin{array}{l}\text { AWMF- } \\
\text { Register- } \\
\text { nummer }\end{array}$ & Stufe & Status & Suizidalität erwähnt \\
\hline $\begin{array}{l}\text { Geschlechtsinkongruenz und Geschlechtsdysphorie im Kindes- } \\
\text { und Jugendalter: Diagnostik und Behandlung }\end{array}$ & 028-014 & S3 & Angemeldet & N.z. \\
\hline Umgang mit Suizidalität & 038-028 & S3 & Angemeldet & Eigene Leitlinie für Suizidalität \\
\hline \multicolumn{5}{|l|}{ „Suizidalität" könnte ggf. berücksichtigt werden } \\
\hline Diagnostik und Behandlung der Rechenstörung & $028-046$ & S3 & Gültig & - \\
\hline $\begin{array}{l}\text { Gutachtliche Untersuchung bei psychischen und psychosomati- } \\
\text { schen Störungen }\end{array}$ & 051-029 & S2k & Gültig & 2-mal erwähnt \\
\hline $\begin{array}{l}\text { Störungen des Sozialverhaltens: Empfehlungen zur Versorgung } \\
\text { und Behandlung }\end{array}$ & $028-020$ & S3 & Gültig bis 09/21 & 2-mal erwähnt \\
\hline $\begin{array}{l}\text { Diagnostik und Behandlung bei der Lese- und/oder Rechtschreib- } \\
\text { störung }\end{array}$ & 028-044 & S3 & In Überarbeitung & - \\
\hline $\begin{array}{l}\text { Sprachentwicklungsstörungen (SES) unter Berücksichtigung um- } \\
\text { schriebener Sprachentwicklungsstörungen (USES), Diagnostik }\end{array}$ & 049-006 & $\mathrm{S} 2 \mathrm{k}$ & $\begin{array}{l}\text { Überarbeitung für } 2021 \\
\text { geplant }\end{array}$ & - \\
\hline
\end{tabular}

se „MAGICapp“1 hilfreich und werden zukünftig zunehmend eingesetzt.

Die Evidenzbasierung ist maßgebend für die wissenschaftliche Legitimation einer Leitlinie, die repräsentative Beteiligung der Anwenderinnen und Anwender sowie die strukturierte Konsensfindung sind dagegen entscheidend für die Akzeptanz und Umsetzung. Auf der Basis von Charakteristika bezüglich der Konsensfindung und systematischer Literaturrecherche werden nach der AWMF 4 Klassen von Leitlinien eingeteilt: S1 (Handlungsempfehlungen von Expertinnen und Experten), S2k (konsensbasiert), S2e (evidenzbasiert) und S3 (evidenz- und konsensbasiert; [34]).

\section{Aktueller Stand der Leitlinien für psychische Erkrankungen in Deutschland}

In Deutschland gibt es eine Leitlinie zur Suizidalität bei Kindern und Jugendlichen, die derzeit noch nicht aktualisiert ist. Des Weiteren gibt es aktuell eine abgelaufene klinische Leitlinie zu nichtsuizidalem, selbstverletzendem Verhalten (NSSV) bei Kindern und Jugendlichen ( $\bullet$ Tab. 1), die eine sinnvolle Ergänzung zur Leitlinie „Suizidalität im Kindes- und Jugendalter" [3] ist. Zudem ist eine Abgrenzung von NSSV und akuter Suizidalität wichtig und sinnvoll.
ZurSuizidalität und zur Suizidprävention im Erwachsenenalter existiert bisher in Deutschland keine eigene Leitlinie. Somit gibt es derzeit keine einzige gültige Leitlinie bezüglich des Umgangs mit Suizidalität und zur Suizidprävention in Deutschland.

Zwar sind in den vergangenen Jahren unter der Federführung der DGPPN und der DGKJP eine Reihe von Leitlinien zu psychischen Erkrankungen erschienen (•Tab. 1) oder auch mit deren Beteiligung (•Tab. 2; siehe auch [35]). Einige dieser Leitlinien haben eigene Kapitel zum Thema suizidales Verhalten. Auffällig ist, dass bei sehr vielen Leitlinien das Wort „Suizid“ bzw. dessen Ableitungen oder Synonyme nicht einmal erwähnt wurden. „Suizidalität“ müsste bzw. sollte nach Ansicht der Autorinnen und Autoren jedoch in den meisten der in den $\bullet$ Tab. 1 und 2 aufgeführten Leitlinien stärker berücksichtigt werden, da die jeweils darin bearbeiteten Störungen nachgewiesene Risikofaktoren für Suizid sind oder - wie aus der klinischen Praxis bekannt ist - oft zusammen mit Erkrankungen vorkommen, die Risikofaktoren für Suizid sind, z. B. Zahnbehandlungsangst bei Abhängigkeitserkrankungen. In diesen Leitlinien sollten zumindest entsprechende Hinweise und Verweise auf die Leitlinien zur „Suizidalität“ erfolgen auch in den Leitlinien, die sich bereits sehr ausführlich der Thematik Suizidalität widmen, wie beispielsweise die Leitlinie zu Autismusspektrumstörungen.

\section{Notwendigkeit einer evidenz- basierten Suizidprävention in Deutschland}

Zwischen 1980 und 2020 kam es zu einer signifikanten Abnahme von Suiziden in Deutschland um mehr als $40 \%$ [1], mutmaßlich unter anderem als Folge einer verbesserten psychiatrisch-psychotherapeutischen Versorgung, eines Ausbaus der niedrigschwelligen Suizidpräventionsangebote (z.B. Krisendienste) und möglicherweise auch von Antistigmabemühungen und konzertierten Aktionen (beispielsweise „Deutsches Bündnis gegen Depression“; [36]). Seither stagnieren die Todesraten durch Suizid auf einem Niveau von ca. 12/100.000 Einwohner. Allerdings ist in den letzten 20 Jahren die Zahl der Todesfälle durch „sonstige oder nicht näher bezeichnete Todesursachen" angestiegen, worunter sich auch Suizide verbergen können. Möglicherweise könnten in Zukunft aufgrund der Einführung der Datenschutzgrundverordnung vom Statistischen Bundesamt vermehrt Fälle in diese Kategorie eingeordnet werden. Da es sich bei Suizid meist um einen prinzipiell verhinderbaren Tod handelt, sind intensivere Maßnahmen 
Tab. 2 AktuellerStand derveröffentlichten Leitlinien im Bereich Psychiatrie unterBeteiligung der DGPPN-und/oderDGKJP (siehe auch [5, 35]), ergänzt um das Ergebnis der Suche nach dem Stichwort "Suizid”

\begin{tabular}{|c|c|c|c|c|}
\hline Leitlinien mit DGPPN- und/oder DGKJP-Beteiligung & $\begin{array}{l}\text { AWMF- } \\
\text { Register- } \\
\text { nummer }\end{array}$ & Stufe & Status & Suizidalität erwähnt \\
\hline \multicolumn{5}{|l|}{ Berücksichtigung der Suizidalität auf jeden Fall erforderlich } \\
\hline $\begin{array}{l}\text { Ärztliche Begutachtung von Menschen mit chronischen Schmer- } \\
\text { zen }\end{array}$ & 094-003 & S3 & Gültig & - \\
\hline $\begin{array}{l}\text { Akute Folgen psychischer Traumatisierung - Diagnostik und Be- } \\
\text { handlung }\end{array}$ & $051-027$ & S2k & Gültig & 8-mal erwähnt \\
\hline Angststörungen & $051-028$ & S3 & Gültig & 34-mal erwähnt \\
\hline Chorea/Morbus Huntington & 030-028 & S2k & Gültig & 3-mal erwähnt \\
\hline $\begin{array}{l}\text { Depression bei Menschen mit Querschnittslähmung: Besonderhei- } \\
\text { ten in der Diagnostik und Behandlung }\end{array}$ & $179-003$ & S1 & Gültig & 5-mal erwähnt \\
\hline $\begin{array}{l}\text { Diagnostik, Therapie und Nachsorge des Hodgkin-Lymphoms bei } \\
\text { erwachsenen Patienten }\end{array}$ & 018-029OL & S3 & Gültig & - \\
\hline $\begin{array}{l}\text { Fibromyalgiesyndrom: Definition, Pathophysiologie, Diagnostik } \\
\text { und Therapie }\end{array}$ & $145-004$ & S3 & Gültig & $\begin{array}{l}\text { 8-mal erwähnt, meist als „Ne- } \\
\text { benwirkung“ von Medikamenten }\end{array}$ \\
\hline Funktionelle Körperbeschwerden & 051-001 & S3 & Gültig & $\begin{array}{l}\text { Exkurs zu: Suizidalität bei funk- } \\
\text { tionellen und somatoformen } \\
\text { Körperbeschwerden }\end{array}$ \\
\hline $\begin{array}{l}\text { Geschlechtsinkongruenz, Geschlechtsdysphorie: Diagnostik, Bera- } \\
\text { tung und Behandlung }\end{array}$ & 138-001 & S3 & Gültig & 12-mal erwähnt \\
\hline $\begin{array}{l}\text { LONTS: Opioide, Langzeitanwendung zur Behandlung bei nicht } \\
\text { tumorbedingten Schmerzen }\end{array}$ & $145-003$ & S3 & Gültig & 3-mal erwähnt \\
\hline $\begin{array}{l}\text { Nicht erholsamer Schlaf/Schlafstörungen - Schlafbezogene At- } \\
\text { mungsstörungen }\end{array}$ & 063-001 & S3 & Gültig & - \\
\hline $\begin{array}{l}\text { Palliativmedizin für Menschen mit einer nicht heilbaren Krebser- } \\
\text { krankung }\end{array}$ & $128-0010 \mathrm{~L}$ & S3 & Gültig & $\begin{array}{l}\text { Kapitel über Todeswunsch und } \\
\text { Suizidalität }\end{array}$ \\
\hline Posttraumatische Belastungsstörung & $155-001$ & S3 & Gültig & 20-mal erwähnt \\
\hline $\begin{array}{l}\text { Schmerzassessment bei älteren Menschen in der vollstationären } \\
\text { Altenhilfe }\end{array}$ & $145-001$ & S3 & Gültig & Einmal erwähnt \\
\hline Visuelle Wahrnehmungsstörungen & $022-020$ & S2k & Gültig & - \\
\hline Zahnbehandlungsangst bei Erwachsenen & 083-020 & S3 & Gültig & - \\
\hline Depression (Nationale Versorgungsleitlinie, NVL) & Nvl-005 & S3 & $\begin{array}{l}\text { In Überarbeitung, Revi- } \\
\text { sion für } 2022 \text { erwartet }\end{array}$ & $\begin{array}{l}\text { Separates Kapitel "Management } \\
\text { bei Suizidgefahr" }\end{array}$ \\
\hline $\begin{array}{l}\text { Diagnostik, Therapie und Verlaufskontrolle des Diabetes mellitus } \\
\text { im Kindes- und Jugendalter }\end{array}$ & 057-016 & S3 & In Überarbeitung & - \\
\hline Parkinson-Syndrom, idiopathisch & 030-010 & S3 & In Überarbeitung & 2-mal erwähnt \\
\hline $\begin{array}{l}\text { Psychoonkologische Diagnostik, Beratung und Behandlung von } \\
\text { erwachsenen Krebspatienten }\end{array}$ & 032-0510L & S3 & In Überarbeitung & 7-mal erwähnt \\
\hline Opioidbezogene Störungen & 076-012 & S3 & Angemeldet & N.z. \\
\hline \multicolumn{5}{|l|}{ "Suizidalität" könnte ggf. berücksichtigt werden } \\
\hline Allgemeine Grundlagen der medizinischen Begutachtung & 094-001 & S2k & Gültig & - \\
\hline Fertilitätserhalt bei onkologischen Erkrankungen & 015-082 & S2k & Gültig & - \\
\hline $\begin{array}{l}\text { Gesundheitliche Aspekte und Gestaltung von Nacht- und Schicht- } \\
\text { arbeit }\end{array}$ & $002-030$ & S2k & Gültig & - \\
\hline Hausärztliche Multimedikation & 053-043 & S3 & Gültig & - \\
\hline Hypersalivation & 017-075 & S2k & Gültig & - \\
\hline Müdigkeit & 053-002 & S3 & Gültig & - \\
\hline Neuroborreliose & 030-071 & S3 & Gültig & - \\
\hline $\begin{array}{l}\text { Operative Behandlung der distalen, mittleren und proximalen } \\
\text { Hypospadie }\end{array}$ & 006-026 & S2k & Gültig & - \\
\hline Peri- und Postmenopause - Diagnostik und Interventionen & 015-062 & S3 & Gültig & - \\
\hline Therapie und Prävention der Adipositas im Kindes- und Jugendal- & 050-002 & S3 & Gültig & - \\
\hline
\end{tabular}




\begin{tabular}{|c|c|c|c|c|}
\hline Leitlinien mit DGPPN- und/oder DGKJP-Beteiligung & $\begin{array}{l}\text { AWMF- } \\
\text { Register- } \\
\text { nummer }\end{array}$ & Stufe & Status & Suizidalität erwähnt \\
\hline Down-Syndrom im Kindes- und Jugendalter & 027-051 & S2k & Gültig bis $07 / 21$ & - \\
\hline Redeflussstörungen, Pathogenese, Diagnostik und Behandlung & 049-013 & S3 & Gültig bis $08 / 21$ & - \\
\hline Chronischer Tinnitus & 017-064 & S3 & In Überarbeitung & - \\
\hline $\begin{array}{l}\text { Depersonalisations-Derealisationssyndrom, Diagnostik und Be- } \\
\text { handlung }\end{array}$ & 051-030 & S2k & In Überarbeitung & - \\
\hline $\begin{array}{l}\text { Epidurale Rückenmarksstimulation zur Therapie chronischer } \\
\text { Schmerzen }\end{array}$ & $008-023$ & S3 & In Überarbeitung & - \\
\hline $\begin{array}{l}\text { Transition von jungen Menschen mit Adipositas von der Pädiatrie } \\
\text { in die Erwachsenenmedizin }\end{array}$ & $050-003$ & S3 & Angemeldet & N.z. \\
\hline \multicolumn{5}{|c|}{$\begin{array}{l}\text { AWMF Arbeitsgemeinschaft der Wissenschaftlichen Medizinischen Fachgesellschaften; DGKJP Deutsche Gesellschaft für Kinder- und Jugendpsychiatrie, Psy } \\
\text { chosomatik und Psychotherapie e. V.; DGPPN Deutsche Gesellschaft für Psychiatrie, Psychotherapie, Psychosomatik und Nervenheilkunde e. V.; N. z. Nicht zU- } \\
\text { treffend; } S 1 \text { Handlungsempfehlungen von Expertinnen und Experten, S2k konsensbasiert, S2e evidenzbasiert, S3 evidenz- und konsensbasiert }\end{array}$} \\
\hline
\end{tabular}

zur Suizidprävention indiziert. Neben dem individuellen Leiden liegt die hohe gesundheitsökonomische Relevanz des Themas auf der Hand. Die Folgekosten für Gesellschaft und Gesundheitssystem sind erheblich [37-39].

Aufgrund der Komplexität des Phänomens Suizidalität ist Suizidprävention eine vielschichtige Aufgabe: Im Bereich des Gesundheitswesens resultiert daraus ein komplexer Hilfebedarf bei der Koordination der unterschiedlichen ambulanten und stationären patientenzentrierten Behandlungsleistungen. Insbesondere aufgrund der Tatsache, dass zumindest teilweise und vorübergehend $\mathrm{Be}$ handlungsmaßnahmen gegen den momentanen Willen der Patientinnen und Patienten eingeleitet werden müssen, ist eine Leitlinie für den Umgang mit Suizidalität im Erwachsenenalter auf höchstem Evidenzniveau dringend notwendig. In solchen Situationen muss die Leitlinie auch über den rein medizinischen Sektor hinausgehen und hat somit eine Relevanz für andere Berufsgruppen wie Einsatzkräfte, gesetzliche und psychosoziale Betreuerinnen und Betreuer, in der Seelsorge Tätige und andere. Eine Übertragung der Inhalte, insbesondere der Empfehlungen der S2k-Leitlinie „Suizidalität im Kindes- und Jugendalter" [3], ist aufgrund altersspezifischer Unterschiede nur eingeschränkt möglich. Dies gilt auch für die damit verbundenen Anforderungen an die Hilfesysteme.

Aktuell werden von Kliniken häufig keine standardisierten Verfahren zum Umgang mit dem Thema „Suizidalität“ verwendet. Einzelne Kliniken setzen selbstentwickelte Standards und Verfahrensanweisungen zum Umgang mit Suizidalität ein. Diesen Empfehlungen und Standards liegt jedoch nicht immer wissenschaftliche Evidenz zugrunde und aus methodischen Gründen handelt es sich nicht um einen Konsens von Fachexpertinnen und -experten unter Einbeziehung von Betroffenen oder Angehörigen. Daraus ergibt sich unmittelbar die Notwendigkeit einer allgemeingültigen, wissenschaftlich fundierten Leitlinie für den Umgang mit Suizidalität in den unterschiedlichen ambulanten wie klinischen Versorgungsfeldern zur Verbesserung der Versorgungsqualität. Eine dezidierte Leitlinie zum Umgang mit suizidalem Verhalten im Erwachsenenalter gibt den beteiligten Akteurinnen und Akteuren der verschiedenen Sektoren eine klare Handlungsanweisung auf Grundlage der vorhandenen Evidenz und verbessert somit die Versorgung von ca. 200.000 von Suizidalität Betroffenen in Deutschland direkt.

Der Nachsorge und der Schnittstelle zum ambulanten Bereich bei Entlassung kommt ebenfalls eine besondere Rolle $\mathrm{zu}$, da die Zeit direkt nach der Entlassung aus der stationären Behandlung eine Zeit des hohen Suizidrisikos ist [40,41]. Klare Angaben zum möglichen Vorgehen bei der Vernetzung und Koordination der verschiedenen Akteure sind ein bedeutsamer Faktor. Durch eine entsprechende Leitlinie kann sowohl die interdisziplinäre (z.B. zwischen Notaufnahme und Psychiatrie oder im Konsiliar- und Liai- sondienst), die intersektorale (z. B. zwischen ambulantem und stationärem Sektor) als auch die Koordination zwischen verschiedenen Berufsgruppen (z. B. dem medizinischen, psychologischen, pflegerischen und sozialarbeiterischen Fachpersonal) besser sichergestellt werden. Durch Konsentierung einer S3-Leitlinie besteht somit ein erhebliches Potenzial für die Verbesserung der Versorgung von betroffenen Patientinnen und Patienten (mit entsprechender Senkung von Mortalität und Morbidität) sowie von deren Angehörigen, auch im Rahmen der Postvention [42].

Auf Basis der beschriebenen Notwendigkeit nationaler Suizidpräventionsprogramme [43] sind die Darstellung und fortlaufende Aktualisierung der vorhandenen wissenschaftlichen Evidenz durch eine Leitlinie ein zentraler Baustein in der Bewertung der möglichen Maßnahmen. Erfreulicherweise wurde Ende August 2021 durch den Innovationsfonds des Gemeinsamen Bundesausschusses (G-BA) die Finanzierung einer S3-Leitlinie „Umgang mit Suizidalität“ bewilligt. Diese Leitlinie ist bei der AWMF angemeldet (- Tab. 1).

Wirkungsvolle Suizidprävention schließt auch Interventionen auf weiteren Handlungsebenen ein: Dazu gehören unter anderem eine umfängliche Erfassung von Daten zu Suizidversuchen und vollendeten Suiziden, die Enttabuisierung der Suizidthematik, eine verantwortungsvolle Berichterstattung über Suizide in den Medien und niedrigschwellige Angebote für Suizidgefährdete sowie ei- 
ne datengeleitete Methodenrestriktion. Suizidprävention zielt auf die Vermeidung und Reduzierung von Suiziden, auf Hilfen für suizidale Personen wie auch auf die Hilfen für Hinterbliebene, die nach einem Suizid ein erhöhtes Risiko für psychische Erkrankungen haben und oft selbst Hilfe und Unterstützung benötigen [33]. Die Verfügbarkeit von Leitlinien ist dabei einer von vielen wichtigen Bausteinen in der Suizidprävention. Die Einrichtung einer unabhängigen zentralen Einrichtung für Suizidprävention zur bundesweiten Datenanalyse, von zentralen Beratungs- und Notfallangeboten (wie beispielsweise einer bundeseinheitlichen Notfallhotline) sowie Öffentlichkeitsarbeit und Multiplikatorschulung sind ebenfalls dringend geboten.

\section{Fazit}

Bisher gibt es im deutschsprachigen Raum zwar eine S2k-Leitlinie zur Suizidalität im Kindes- und Jugendalter [3], jedoch keine Leitlinie zum Umgang mit Suizidalität und zur Suizidprävention im Erwachsenenalter. Die Fertigstellung einer S3-Leitlinie zum Umgang mit Suizidalität im Erwachsenenalter wird jedoch für 2024 erwartet. In einer S3-Leitlinie können Handlungsempfehlungen für die in den verschiedenen Sektoren des Gesundheitssystems Tätigen formuliert werden. Leitlinien zur Suizidalität und zur Suizidprävention tragen erheblich $\mathrm{zu}$ einer besseren Wissensvermittlung sowie zur Implementation vorhandener Evidenz in der Versorgung von Suizidgefährdeten und betroffenen Angehörigen bei, insbesondere auch in den nichtpsychiatrisch-psychotherapeutischen Fachgebieten. Basierend auf der wissenschaftlichen Evidenz, einem interdisziplinären Konsens zur Prävention suizidalen Verhaltens und der klinischen Behandlung suizidgefährdeter Personen können Standards für die Früherkennung, Klassifikation, Diagnostik, Therapie und Nachsorge geschaffen werden.

Bei Verdacht auf das Vorliegen einer psychischen Störung kann/sollte in der Leitlinie „Umgang mit Suizidalität“ auf die Leitlinien der jeweiligen psychischen Erkrankung bzw. Störung hingewiesen werden. Damit wird die indivi- duelle, direkte Versorgung der betroffenen Patientinnen und Patienten optimiert und die Struktur- und Prozessqualität der mit Suizidalität befassten Einrichtungen des Gesundheitssystems verbessert, einschließlich an deren Schnittstellen zwischen ambulanter und stationärer Versorgung sowie zwischen einzelnen medizinischen Fachgebieten. Ein standardisiertes Vorgehen im Hinblick auf Erfassung von und Umgang mit Suizidalität ist daher ein wesentlicher Beitrag zur Patientensicherheit. Die Erstellung einer Patientenleitlinie, mithilfe derer sich sowohl Betroffene als auch Angehörige bzw. Hinterbliebene informieren können, ist darüber hinaus geplant.

\section{Korrespondenzadresse}

Prof. Dr. Barbara Schneider, M. Sc. MHBA Abteilung für Abhängigkeitserkrankungen, Psychiatrie und Psychotherapie, LVR-Klinik Köln Wilhelm-Griesinger-Str. 23, 51109 Köln, Deutschland

B.Schneider@lvr.de

Förderung. Die S3-Leitlinie „Umgang mit Suizidalität" unter Projektleitung von A. Reif (Stellvertretung B. Schneider) wird vom Innovationsfond des Gemeinsamen Bundesausschusses gefördert (FK 01VFS21017 LL - UmS).

Funding. Open Access funding enabled and organized by Projekt DEAL.

\section{Einhaltung ethischer Richtlinien}

Interessenkonflikt. B. Schneider, A. Reif, B. Wagner und M. Wolfersdorf geben an, dass kein Interessenkonflikt besteht. B. Schneider und B. Wagner gehören der ehrenamtlich arbeitenden geschäftsführenden Leitung des Nationalen Suizidpräventionsprogramms für Deutschland an. A. Reif ist Mitglied der Steuerungsgruppe der NVL Unipolare Depression sowie Vorstandsmitglied der DGPPN und im Stiftungsrat der Stiftung Deutsche Depressionshilfe. Er erhält Sprecherhonorare bzw. ist beratend tätig für Janssen, Medice, Shire/Takeda und SAGE.

Für diesen Beitrag wurden von den Autoren keine Studien an Menschen oder Tieren durchgeführt. Für die aufgeführten Studien gelten die jeweils dort angegebenen ethischen Richtlinien.

Open Access. Dieser Artikel wird unter der Creative Commons Namensnennung 4.0 International Lizenz veröffentlicht, welche die Nutzung, Vervielfältigung, Bearbeitung, Verbreitung und Wiedergabe in jeglichem Medium und Format erlaubt, sofern Sie den/die ursprünglichen Autor(en) und die Quelle ordnungsgemäß nennen, einen Link zur Creative Commons Lizenz beifügen und angeben, ob Änderungen vorgenommen wurden.

Die in diesem Artikel enthaltenen Bilder und sonstiges Drittmaterial unterliegen ebenfalls der genannten Creative Commons Lizenz, sofern sich aus der Abbildungslegende nichts anderes ergibt. Sofern das betreffende Material nicht unter der genannten Creative Commons Lizenz steht und die betreffende Handlung nicht nach gesetzlichen Vorschriften erlaubt ist, ist für die oben aufgeführten Weiterverwendungen des Materials die Einwilligung des jeweiligen Rechteinhabers einzuholen.

Weitere Details zur Lizenz entnehmen Sie bitte der Lizenzinformation auf http://creativecommons.org/ licenses/by/4.0/deed.de.

\section{Literatur}

1. Statistisches Bundesamt Deutschland (2021) Sterbefälle nach äußeren Ursachen und ihren Folgen (ab 1998). www.gbe-bund.de.Zugegriffen: 2. März 2021

2. Berman AL (2011) Estimating the population of survivors of suicide: seeking an evidence base. Suicide Life Threat Behav 41:110-116. https://doi. org/10.1111/j.1943-278X.2010.00009.x

3. Arbeitsgemeinschaft der Wissenschaftlichen Medizinischen Fachgesellschaften (AWMF) (2016) Suizidalität im Kindes- und Jugendalter. https:// www.awmf.org/leitlinien/detail/ll/028-031.html. Zugegriffen: 27.Sept. 2021

4. National Institute for Health and Care Excellence (2018) Preventing suicide in community and custodial settings. NICEguideline [NG105]. https:// www.nice.org.uk/guidance/NG105. Zugegriffen: 27. Sept. 2021

5. Arbeitsgemeinschaft der Wissenschaftlichen Medizinischen Fachgesellschaften (AWMF) (2021) https://www.awmf.org/leitlinien/awmfregelwerk/ll-entwicklung.html. Zugegriffen: 27. Sept. 2021

6. National Institute for Health and Care Excellence NICE (2014) https://www.nice.org.uk/process/ pmg20/chapter/introduction. Zugegriffen: 27. Sept. 2021 (last updated 2020)

7. World Health Organization (2014) Suicide prevention: A global imperative. https://www.who.int/ publications/i/item/9789241564779.Zugegriffen: 16. Sept. 2014

8. Turecki G, Brent DA (2016) Suicide and suicidal behaviour. Lancet 387:1227-1239. https://doi. org/10.1016/S0140-6736(15)00234-2

9. Fazel S, Runeson B (2020) Suicide. N Engl J Med 382:266-274. https://doi.org/10.1056/ NEJMra1902944

10. Hawton K, Bergen H, Cooper J et al (2015) Suicide following self-harm: findings from the Multicentre Study of self-harm in England, 2000-2012. J Affect Disord 175:147-151. https://doi.org/10.1016/j. jad.2014.12.062

11. Olfson M, Wall M, Wang S, Crystal S, Gerhard T, Blanco C (2017) Suicide following deliberate selfharm. Am J Psychiatry 174:765-774. https://doi. org/10.1176/appi.ajp.2017.16111288

12. Haserück A, Richter-Kuhlmann E (2021) Dtsch Arztebl 118:A-863 /B-719

13. Oregon Health Authority (2019) https:// www.arcgis.com/apps/MapSeries/index.html? appid=9c59be59ef7142dfad40d95e3b36f588. Zugegriffen: 14.Apr. 2019 


\section{Leitthema}

14. World Health Organization (2020) Global Health Estimates 2019: deaths by cause, age, sex, by country and by region, 2000-2019. World Health Organízation, Genf, Geneva

15. Schneider B (2003) Risikofaktoren für Suizid. Roderer, Regensburg

16. Marttunen MJ, Aro HM, Henriksson MM, Lönnqvist JK (1991) Mental disorders in adolescent suicide. DSM-III-R axes I and II diagnoses in suicides among 13- to 19-year-olds in Finland. Arch Gen Psychiatry 48:834-839

17. Moitra M, Santomauro D, Degenhardt Let al (2021) Estimating the risk of suicide associated with mental disorders: A systematic review and metaregression analysis. J Psychiatr Res 137:242-249. https://doi.org/10.1016/j.jpsychires.2021.02.053

18. Harris EC, Barraclough B (1997) Suicide as an outcome for mental disorders. Br J Psychiatry 170:205-228

19. Harris EC, Barraclough B (1998) Excess mortality of mental disorder. Br J Psychiatry 173:11-53

20. Wilcox HC, Conner KR, Caine ED (2004) Association of alcohol and drug use disorders and completed suicide: an empirical review of cohort studies. Drug Alcohol Depend 76(Suppl):S11-S19. https://doi. org/10.1016/j.drugalcdep.2004.08.003

21. Liu RT, Steele SJ, Hamilton JL et al (2020) Sleep and suicide: A systematic review and meta-analysis of longitudinal studies. Clin Psychol Rev 81:101895. https://doi.org/10.1016/j.cpr.2020.101895

22. Crump C, Sundquist K, Sundquist J, Winkleby MA (2014) Sociodemographic, psychiatric and somatic risk factors for suicide: a Swedish national cohort study. Psychol Med 44:279-289. https://doi.org/ $10.1017 /$ S0033291713000810

23. Conner KR, Beautrais AL, Conwell Y (2003) Moderators of the relationship between alcohol dependence and suicide and medically serious suicide attempts: analyses of Canterbury Suicide Project data. Alcohol Clin Exp Res 27:1156-1161. https:// doi.org/10.1097/01.ALC.0000075820.65197.FD

24. Erlangsen A, Stenager E, Conwell $Y$ et al (2020) Association between neurological disorders and death by suicide in Denmark. JAMA 323:444-454. https://doi.org/10.1001/jama.2019.21834

25. Du L, Shi HY, Yu HR et al (2020) Incidence of suicide death in patients with cancer: A systematic review and meta-analysis. J Affect Disord 276:711-719. https://doi.org/10.1016/j.jad.2020.07.082

26. Pompili M, Venturini P, Montebovi F et al (2013) Suicide risk in dialysis: review of current literature. Int J Psychiatry Med 46:85-108. https://doi.org/10. 2190/PM.46.1.f

27. Chen X, Zhang H, Xiao G, Lv C (2020) Prevalence of suicidal ideation among stroke survivors: $A$ systematic review and meta-analysis. Top Stroke Rehabil. https://doi.org/10.1080/10749357.2020. 1846933

28. Zaorsky NG, Zhang Y, Tuanquin L, Bluethmann SM, ParkHS, Chinchilli VM (2019) Suicide among cancer patients. Nat Commun 10:207. https://doi.org/10. 1038/s41467-018-08170-1

29. Erlangsen A, Drefahl S, Haas A, Bjorkenstam C, Nordentoft M, Andersson G (2020) Suicide among persons who entered same-sex and opposite-sex marriage in Denmark and Sweden, 1989-2016: a binational, register-based cohort study. J Epidemiol Community Health 74:78-83. https://doi.org/10.1136/jech-2019-213009

30. Plöderl M, Tremblay P (2015) Mental health of sexual minorities. A systematic review. Int Rev Psychiatry 27:367-385. https://doi.org/10.3109/ 09540261.2015.1083949
31. Frisch M, Simonsen J (2013) Marriage, cohabitation and mortality in Denmark: national cohort study of 6.5 million persons followed for up to three decades (1982-2011). Int J Epidemiol 42:559-578. https://doi.org/10.1093/ije/dyt024

32. Amiri S (2020) Prevalence of suicide in immigrants/ refugees: a systematic review and meta-analysis. Arch Suicide Res 1080:1802379. https://doi.org/ 10.1080/13811118.2020.1802379:1-36

33. Qin P, Agerbo E, Mortensen PB (2002) Suicide risk in relation to family history of completed suicide and psychiatric disorders: a nested case-control study based on longitudinal registers. Lancet 360:1126-1130. https://doi.org/10.1016/S01406736(02)11197-4

34. Nationale Versorgungsleitlinien NVL (2021) https://www.leitlinien.de/hintergrund/ leitliniengrundlagen. Zugegriffen: 27 . Sept. 2021

35. Voderholzer U, Hohagen F (2021) Stand der Leitlinien. In: Therapie psychischer Erkrankungen Urban \& Fischer, München, Jena

36. Zalsman G, Hawton K, Wasserman D et al (2016) Suicide prevention strategies revisited: 10-year systematic review. Lancet Psychiatry 3:646-659. https://doi.org/10.1016/S2215-0366(16)30030-X

37. Shepard DS, Gurewich D, Lwin AK, Reed GA Jr., Silverman MM (2016) Suicideand suicidal attempts in the United States: costs and policy implications. Suicide Life Threat Behav 46:352-362. https://doi. org/10.1111/sltb.12225

38. Wolfersdorf M, Martinez C (1998) Suizid bei Depression, verlorene Lebensjahre und Bruttosozialprodukt. Was bringt Suizidprävention? [Suicide in depression, lost years of life and gross social consequences. What is the outcome of suicide prevention?]. Psychiatr Prax 25:139-141

39. Lyszczarz B (2021) Production losses attributable to suicide deaths in European Union. BMC Public Health 21:950. https://doi.org/10.1186/s12889021-11010-5

40. Hoffmann-Richter U, Kanzig S, Frei A, Finzen A (2002) Suicide after discharge from psychiatric hospital. Psychiatr Prax 29:22-24. https://doi.org/ 10.1055/s-2002-19674

41. Sakinofsky I (2014) Preventing suicide among inpatients. Can J Psychiatry 59:131-140. https:// doi.org/10.1177/070674371405900304

42. Schmidtke A, Schaller S (2012) Postvention bei suizidalen Handlungen. Psychother Dialog 13:50-54. https://doi.org/10.1055/s-0032-1304978

43. Zalsman G, Hawton K, Wasserman D et al (2017) Evidence-based national suicide prevention taskforce in Europe: A consensus position paper. Eur Neuropsychopharmacol 27:418-421. https:// doi.org/10.1016/j.euroneuro.2017.01.012 